\title{
NEVANLINNA'S FIVE-VALUE THEOREM FOR ALGEBROID FUNCTIONS
}

\author{
ASHOK RATHOD
}

\begin{abstract}
By using the second main theorem of the algebroid function, we study the following problem. Let $W_{1}(z)$ and $W_{2}(z)$ be two $\nu$-valued non-constant algebroid functions, $a_{j}(j=1,2, \ldots, q)$ be $q \geqslant 4 \nu+1$ distinct complex numbers or $\infty$. Suppose that $k_{1} \geqslant k_{2} \geqslant \ldots \geqslant k_{q}, m$ are positive integers or $\infty, 1 \leqslant m \leqslant q$ and $\delta_{j} \geqslant 0, j=1,2, \ldots, q$, are such that

$$
\left(1+\frac{1}{k_{m}}\right) \sum_{j=m}^{q} \frac{1}{1+k_{j}}+3 \nu+\sum_{j=1}^{q} \delta_{j}<(q-m-1)\left(1+\frac{1}{k_{m}}\right)+m .
$$

Let $B_{j}=\bar{E}_{k_{j}}\left(a_{j}, f\right) \backslash \bar{E}_{k_{j}}\left(a_{j}, g\right)$ for $j=1,2, \ldots, q$. If

$$
\bar{N}_{B_{j}}\left(r, \frac{1}{W_{1}-a_{j}}\right) \leqslant \delta_{j} T\left(r, W_{1}\right)
$$

and

$$
\liminf _{r \rightarrow \infty} \frac{\sum_{j=1}^{q} \bar{N}_{k_{j}}\left(r, \frac{1}{W_{1}-a_{j}}\right)}{\sum_{j=1}^{q} \bar{N}_{k_{j}}\left(r, \frac{1}{W_{2}-a_{j}}\right)}>\frac{\nu k_{m}}{\left(1+k_{m}\right) \sum_{j=1}^{q} \frac{k_{j}}{k_{j}+1}-2 \nu\left(1+k_{m}\right)+\left(m-2 \nu-\sum_{j=1}^{q} \delta_{j}\right) k_{m}},
$$
\end{abstract}

then $W_{1}(z) \equiv W_{2}(z)$. This result improves and generalizes the previous results given by Xuan and Gao.

Keywords: value distribution theory, Nevanlinna theory, algebroid functions, uniqueness.

Subject Classification: 30D35

\section{INTRODUCTION}

The value distribution theory of meromorphic functions was extended to the corresponding theory of algebroid functions by Ullarich [1] and Valiron [2] around 1930, and important results on uniqueness for algebroid functions were obtained. It is well known that Valiron obtained a famous $(4 \nu+1)$-valued theorem. The uniqueness theory of algebroid functions is an interesting problem in the value distribution theory. Many researchers like Valiron [2], Baganas [3], He [4] and others ([6],[7],[9-27]) made lot of work in this area. In this article, we extend a result by Indrajit Lahiri and Rupa Pal [5] in the Nevanlinna's value distribution theory of meromorphic functions on Nevanlinna's five values theorem to algebroid functions

Let $A_{\nu}(z), A_{\nu-1}(z), \ldots, A_{0}(z)$ be analytic functions with no common zeros in the complex plane and consider the equation

$$
A_{\nu}(z) W^{\nu}+A_{\nu-1}(z) W^{\nu-1}+\ldots+A_{1}(z) W+A_{0}(z)=0 .
$$

Ashok Rathod, Nevanlinna's five-value theorem for algebroid functions.

(C)ASHOK RATHOD 2018 .

The author is supported by the UGC-Rajiv Gandhi National Fellowship (no. F1-17.1/2013-14-SC-KAR40380) of India.

Submitted April 06, $201 \%$. 
This equation defines a $\nu$-valued algebroid function $W(z)$ [8].

It is well known [8] that on the complex plane with the projection of the critical points of the function $W$ cut out, the Nevanlinna characteristic $T(r, W)$ is defined as

$$
T(r, W)=m(r, W)+N(r, W),
$$

where

$$
\begin{aligned}
& m(r, W)=\frac{1}{2 \pi \nu} \sum_{j=1}^{\nu} \int_{0}^{2 \pi} \log ^{+}\left|w_{j}\left(r e^{i \theta}\right)\right| d \theta, \\
& N(r, W)=\frac{1}{\nu} \int_{0}^{r} \frac{n(t, W)-n(0, W)}{t} d t+\frac{n(0, W)}{\nu} \log r .
\end{aligned}
$$

Let $w_{i}(z)$ and $m_{j}(z)$ be one-valued branches of two ( $\mu$-valued and $\nu$-valued) algebroid functions. Following Prokopovich [15], we consider their quotient in the domain of the complex plane with the projection of the critical points of both functions cut out. The one-valued branches of th function $W / M(W \cdot M)$ are defined as $w_{i} / m_{j}\left(w_{i} \cdot m_{j}\right)$, where $1 \leqslant i \leqslant m$, $1 \leqslant j \leqslant n$. The Nevanlinna's characteristic $T(r, W / M)$ is defined by $T(r, W)+T(r, M)$.

Lemma 1 (8). Let $W(z)$ be a $\nu$-valued algebroid function and $\left\{a_{j}\right\}_{j=1}^{q} \subset \overline{\mathbb{C}}$ be $q$ distinct complex numbers and let $\left\{k_{j}\right\}_{j=1}^{q} \subset \mathbb{N}$ be q positive integers. Then

$$
\begin{array}{r}
(q-2 \nu) T(r, W) \leqslant \sum_{k=1}^{q} \frac{k_{j}}{k_{j}+1} \bar{N}_{\left.k_{j}\right)}\left(r, W=a_{j}\right)+\sum_{k=1}^{q} \frac{1}{k_{j}+1} N\left(r, W=a_{j}\right)+S(r, W), \\
\left(q-2 \nu-\sum_{k=1}^{q} \frac{1}{k_{j}+1}\right) T(r, W) \leqslant \sum_{k=1}^{q} \frac{k_{j}}{k_{j}+1} \bar{N}_{\left.k_{j}\right)}\left(r, W=a_{j}\right)+N\left(r, W=a_{j}\right)+S(r, W) .
\end{array}
$$

In 2006 Zu-Xing Xuan and Zong-Sheng Gao [18] improved this statement as follows.

Theorem 1. Let $W(z)$ and $M(z)$ be two $\nu$-valued non-constant algebroid functions, let $a_{j}(j=1,2, \ldots, 4 \nu+1)$ be $4 \nu+1$ distinct complex numbers in $\overline{\mathbb{C}}$. If

$$
\bar{E}_{2 \nu+1)}\left(a_{j}, W\right)=\bar{E}_{2 \nu+1)}\left(a_{j}, M\right), \quad j=1,2, \ldots, 2 \nu+1
$$

and

$$
\bar{E}_{2 \nu)}\left(a_{j}, W\right)=\bar{E}_{2 \nu)}\left(a_{j}, M\right), \quad j=1,2, \ldots, 4 \nu+1,
$$

then $W(z)=M(z)$

\section{MAIn RESUlts}

Let $W(z)$ be a $\nu$-valued algebroid function and $a \in \overline{\mathbb{C}}$ be a complex number. The symbol $\bar{E}_{k}(W=a)$ denotes the set of zeros of $W(z)-a$, whose multiplicities are not greater than $\mathrm{k}$. The symbol $\bar{n}_{k)}(W=a)$ stands for the number of distinct zeros of $W(z)-a$ in $|z| \leqslant r$, whose multiplicities do not exceed $k$ and are counted only once. Similarly, we define the functions $\bar{n}_{(k+1}(r, W=a), \bar{N}_{k)}(r, W=a)$ and $\bar{N}_{(k+1}(r, W=a)$.

In this paper, we study the problem on the Nevanlinna's five value theorem for algebroid functions. To state our main theorem, we first introduce the following definition.

Definition 1. For $B \subset \mathbb{A}$ and $a \in \overline{\mathbb{C}}$, we denote by $\bar{N}_{B}\left(r, \frac{1}{f-a}\right)$ the reduced counting function of the zeros of $f-a$ on $\mathbb{A}$ belonging to the set $B$.

Theorem 2. Let $W_{1}(z)$ and $W_{2}(z)$ be two $\nu$-valued non-constant algebroid functions, let $a_{j}(j=1,2, \ldots, q)$ be $q \geqslant 4 \nu+1$ distinct complex numbers or $\infty$. Suppose that 
$k_{1} \geqslant k_{2} \geqslant \ldots \geqslant k_{q}, m$ are positive integers or $\infty ; 1 \leqslant m \leqslant q$ and $\delta_{j} \geqslant 0, j=1,2, \ldots, q$, are such that

$$
\left(1+\frac{1}{k_{m}}\right) \sum_{j=m}^{q} \frac{1}{1+k_{j}}+3 \nu+\sum_{j=1}^{q} \delta_{j}<(q-m-1)\left(1+\frac{1}{k_{m}}\right)+m .
$$

Let $B_{j}=\bar{E}_{k_{j}}\left(a_{j}, f\right) \backslash \bar{E}_{k_{j}}\left(a_{j}, g\right)$ for $j=1,2, \ldots, q$. If

$$
\bar{N}_{B_{j}}\left(r, \frac{1}{W_{1}-a_{j}}\right) \leqslant \delta_{j} T\left(r, W_{1}\right)
$$

and

$$
\liminf _{r \rightarrow \infty} \frac{\sum_{j=1}^{q} \bar{N}_{k_{j}}\left(r, \frac{1}{W_{1}-a_{j}}\right)}{\sum_{j=1}^{q} \bar{N}_{k_{j}}\left(r, \frac{1}{W_{2}-a_{j}}\right)}>\frac{\nu k_{m}}{\left(1+k_{m}\right) \sum_{j=1}^{q} \frac{k_{j}}{k_{j}+1}-2 \nu\left(1+k_{m}\right)+\left(m-2 \nu-\sum_{j=1}^{q} \delta_{j}\right) k_{m}}
$$

then $W_{1}(z) \equiv W_{2}(z)$.

Proof. Suppose that $W_{1}(z) \neq W_{2}(z)$. The by Lemma 1 for each integer $m, 1 \leqslant m \leqslant q$, we have

$$
\begin{aligned}
(q-2 \nu) T\left(r, W_{1}\right) & \leqslant \sum_{j=1}^{q} \bar{N}\left(r, \frac{1}{W_{1}-a_{j}}\right)+S\left(r, W_{1}\right) \\
& \leqslant \sum_{j=1}^{q}\left\{\bar{N}_{\left.k_{j}\right)}\left(r, \frac{1}{W_{1}-a_{j}}\right)+\bar{N}_{\left(k_{j+1}\right.}\left(r, \frac{1}{W_{1}-a_{j}}\right)\right\}+S\left(r, W_{1}\right) \\
& \leqslant \sum_{j=1}^{q}\left\{\bar{N}_{\left.k_{j}\right)}\left(r, \frac{1}{W_{1}-a_{j}}\right)+\frac{1}{1+k_{j}} N_{\left(k_{j+1}\right.}\left(r, \frac{1}{W_{1}-a_{j}}\right)\right\}+S\left(r, W_{1}\right) \\
& \leqslant \sum_{j=1}^{q}\left\{\frac{k_{j}}{1+k_{j}} \bar{N}_{\left.k_{j}\right)}\left(r, \frac{1}{W_{1}-a_{j}}\right)+\frac{1}{1+k_{j}} N\left(r, \frac{1}{W_{1}-a_{j}}\right)\right\}+S\left(r, W_{1}\right) \\
& \leqslant \sum_{j=1}^{q} \frac{k_{j}}{1+k_{j}} \bar{N}_{\left.k_{j}\right)}\left(r, \frac{1}{W_{1}-a_{j}}\right)+\sum_{j=1}^{q} \frac{1}{1+k_{j}} T\left(r, W_{1}\right)+S\left(r, W_{1}\right) \\
& \leqslant \sum_{j=1}^{q}\left(\frac{k_{j}}{1+k_{j}}-\frac{k_{m}}{1+k_{m}}\right) \bar{N}_{\left.k_{j}\right)}\left(r, \frac{1}{W_{1}-a_{j}}\right)+\sum_{j=1}^{q} \frac{1}{1+k_{j}} T\left(r, W_{1}\right) \\
& +\sum_{j=1}^{q} \frac{k_{m}}{1+k_{m}} \bar{N}_{\left.k_{j}\right)}\left(r, \frac{1}{W_{1}-a_{j}}\right)+S\left(r, W_{1}\right) \\
& \leqslant \sum_{j=1}^{q} \frac{k_{m}}{1+k_{m}} \bar{N}_{\left.k_{j}\right)}\left(r, \frac{1}{W_{1}-a_{j}}\right) \\
& +\left(m-1-\frac{(m-1) k_{m}}{k_{m}+1}+\sum_{j=1}^{q} \frac{k_{j}}{1+k_{j}}\right) T\left(r, W_{1}\right)+S\left(r, W_{1}\right) \\
& \left(m-{ }_{1}\right)
\end{aligned}
$$

Therefore

$$
\left.\left(\sum_{j=m}^{q} \frac{k_{j}}{k_{j}+1}-2 \nu+\frac{(m-1) k_{m}}{k_{m}+1}\right) T\left(r, W_{1}\right) \leqslant \sum_{j=1}^{q} \frac{k_{m}}{k_{m}+1} \bar{N}_{k_{j}}\right)\left(r, \frac{1}{W_{1}-a_{j}}\right)+S\left(r, W_{1}\right) .
$$


Similarly,

$$
\left.\left(\sum_{j=m}^{q} \frac{k_{j}}{k_{j}+1}-2 \nu+\frac{(m-1) k_{m}}{k_{m}+1}\right) T\left(r, W_{2}\right) \leqslant \sum_{j=1}^{q} \frac{k_{m}}{k_{m}+1} \bar{N}_{k_{j}}\right)\left(r, \frac{1}{W_{2}-a_{j}}\right)+S\left(r, W_{2}\right) .
$$

Since $B_{j}=\bar{E}_{\left.k_{j}\right)}\left(a_{j}, W_{1}\right) \bar{E}_{\left.k_{j}\right)}\left(a_{j}, W_{2}\right)$, let $D_{j}=\bar{E}_{k_{j}}\left(a_{j}, W_{1}\right) B_{j}$ for $j=1,2, \ldots, q$. Thus, by (5) and (6), for a sequence of values of $r$ tending to $\infty$ we get:

$$
\begin{aligned}
\sum_{j=m}^{q} \bar{N}_{k_{j}}\left(r, \frac{1}{W_{1}-a_{j}}\right)= & \sum_{j=m}^{q} \bar{N}_{B_{j}}\left(r, \frac{1}{W_{1}-a_{j}}\right)+\sum_{j=m}^{q} \bar{N}_{\left.D_{j}\right)}\left(r, \frac{1}{W_{1}-a_{j}}\right) \\
& \leqslant \sum_{j=m}^{q} \delta_{j} T\left(r, W_{1}\right)+\nu N\left(r, \frac{1}{W_{1}-W_{2}}\right) \\
& \leqslant\left(\nu+\sum_{j=m}^{q} \delta_{j}\right) T\left(r, W_{1}\right)+\nu T\left(r, W_{2}\right)+O(1)
\end{aligned}
$$

Therefore,

$$
\begin{gathered}
\left.\left(\sum_{j=m}^{q} \frac{k_{j}}{k_{j}+1}-2 \nu+\frac{(m-1) k_{m}}{k_{m}+1}+O(1)\right) \bar{N}_{k_{j}}\right)\left(r, \frac{1}{W_{1}-a_{j}}\right) \\
\leqslant\left(\nu+\sum_{j=m}^{q} \delta_{j}\right) \sum_{j=1}^{q} \frac{k_{m}}{k_{m}+1} \bar{N}_{\left.k_{j}\right)}\left(r, \frac{1}{W_{1}-a_{j}}\right) \\
\quad+(\nu+O(1)) \sum_{j=1}^{q} \frac{k_{m}}{k_{m}+1} \bar{N}_{k_{j}}\left(r, \frac{1}{W_{2}-a_{j}}\right) .
\end{gathered}
$$

Since

$$
1 \geqslant \frac{k_{1}}{k_{1}+1} \geqslant \ldots \geqslant \frac{k_{q}}{k_{q}+1} \geqslant \frac{1}{2}
$$

by (7), for a sequence of values of $r$ tending to $+\infty$, we get

$$
\begin{aligned}
& \left.\left(\sum_{j=m}^{q} \frac{k_{j}}{k_{j}+1}-2 \nu+\frac{(m-1) k_{m}}{k_{m}+1}-\frac{k_{m}}{k_{m}+1}\left(\nu+\sum_{j=m}^{q} \delta_{j}\right)+O(1)\right) \bar{N}_{k_{j}}\right)\left(r, \frac{1}{W_{1}-a_{j}}\right) \\
& \leqslant(\nu+O(1)) \frac{k_{m}}{k_{m}+1} \sum_{j=1}^{q} \bar{N}_{k_{j}}\left(r, \frac{1}{W_{2}-a_{j}}\right) .
\end{aligned}
$$

This implies that

$$
\begin{aligned}
\liminf _{r \rightarrow \infty} \frac{\bar{N}_{k_{j}}\left(r, \frac{1}{W_{1}-a_{j}}\right)}{\bar{N}_{k_{j}}\left(r, \frac{1}{W_{2}-a_{j}}\right)} & \leqslant \frac{\nu \frac{k_{m}}{k_{m}+1}}{\left(\sum_{j=m}^{q} \frac{k_{j}}{k_{j}+1}-2 \nu+\frac{k_{m}}{k_{m}+1}\left(m-2 \nu-\sum_{j=m}^{q} \delta_{j}\right)\right)}, \\
& \leqslant \frac{\nu k_{m}}{\left(1+k_{m}\right)\left(\sum_{j=m}^{q} \frac{k_{j}}{k_{j}+1}-2 \nu\left(1+k_{m}\right)+k_{m}\left(m-2 \nu-\sum_{j=m}^{q} \delta_{j}\right)\right)} .
\end{aligned}
$$

This i contradicts equation (2). Thus, we have $f(z) \not \equiv g(z)$. The proof is complete.

Theorem 2 yield the following corollaries. 
Corollary 1. Let $m=1, k_{j}=\infty$ for $j=1,2,3, \ldots, q$ and

$$
\gamma=\liminf _{r \rightarrow \infty} \frac{\bar{N}_{\left.k_{j}\right)}\left(r, \frac{1}{W_{1}-a_{j}}\right)}{\bar{N}_{\left.k_{j}\right)}\left(r, \frac{1}{W_{2}-a_{j}}\right)}>\frac{1}{q-2 \nu+1}
$$

If $\bar{N}_{B_{j}}\left(r, \frac{1}{W_{1}-a_{j}}\right) \leqslant \delta_{j} T\left(r, W_{1}\right)$, where $\delta \geqslant 0$ satisfies

$$
0 \leqslant \sum_{j=1}^{q} \delta_{j}<k-(2 \nu+1)-\frac{1}{\gamma}
$$

then $f(z) \equiv g(z)$

If we take $q=4 \nu+1$ and $\bar{E}\left(a_{j}, f\right) \subseteq \bar{E}\left(a_{j}, f\right)$, then $B_{j}=\emptyset$ for $j=1,2, \ldots, 4 \nu+1$. Therefore, if we choose $\delta_{j}=0$ for $j=1,2, \ldots, 4 \nu+1$ and take any constant $\gamma$ obeying $0 \leqslant 2 \nu-\frac{1}{\gamma}$ in Corollary 1 , we can get that $f \equiv g$. Moreover, if $q=4 \nu+1$ and $\bar{E}\left(a_{j}, f\right)=\bar{E}\left(a_{j}, g\right)$, then $\gamma=1$ and $\delta_{j}=0$ for $j=1,2, \ldots, 4 \nu+1$; this implies $f \equiv g$. Then Corollary 1 is an improvement of Theorem 1.

Corollary 2. Let $W_{1}(z)$ and $W_{2}(z)$ be two $\nu$-valued non-constant algebroid functions, let $a_{j}$, $j=1,2, \ldots, q$, be $q \geqslant 5$ distinct complex numbers or $\infty$. Suppose that $k_{1}, k_{2}, \ldots, k_{q}$ are positive integers or $\infty$ with $k_{1} \geqslant k_{2} \geqslant \ldots \geqslant k_{q}$ if $\bar{E}_{\left.k_{j}\right)}\left(a_{j}, f\right) \subseteq \bar{E}_{\left.k_{j}\right)}\left(a_{j}, g\right)$ and

$$
\sum_{j=2 \nu}^{q} \frac{k_{j}}{k_{j}+1}-\frac{k_{1}}{\gamma\left(k_{1}+1\right)}-2 \nu>0,
$$

where $\gamma$ is as stated in Corollary 1. Then $f(z) \equiv g(z)$.

Corollary 3. Under the assumptions of Corollary 2, we have $\bar{E}_{\left.k_{j}\right)}\left(a_{j}, W_{1}\right)=\bar{E}_{k_{j}}\left(a_{j}, W_{2}\right)$ and

$$
\sum_{j=2 \nu}^{q} \frac{k_{j}}{k_{j}+1}-\frac{k_{1}}{\left(k_{1}+1\right)}-2 \nu>0
$$

Corollary 4. Let $W_{1}(z)$ and $W_{2}(z)$ be two $\nu$-valued non-constant algebroid functions, let $a_{j}$, $j=1,2, \ldots, q$, be $q \geqslant 5$ distinct complex numbers or $\infty$. Suppose that $k_{1}, k_{2}, \ldots, k_{q}$ are positive integers or $\infty$ with $k_{1} \geqslant k_{2} \geqslant \ldots \geqslant k_{q}$ if $\bar{E}_{k_{j}}\left(a_{j}, f\right) \subseteq \bar{E}_{\left.k_{j}\right)}\left(a_{j}, g\right)$ and

$$
\sum_{j=2 \nu}^{q} \frac{k_{j}}{k_{j}+1}-2 \nu+\frac{\left(m-2 \nu-\frac{1}{\gamma}\right) k_{m}}{\gamma\left(k_{m}+1\right)}-2 \nu>0,
$$

where $\gamma$ is as stated a in Corollary 1. Then $f(z) \equiv g(z)$.

\section{BIBLIOGRAPHY}

1. E. Ullrich. Über den Einfluß der verzweigtheit einer algebloide auf ihre wertvertellung // J. Reine Angew. Math. 1932:167, 198-220 (1932).

2. G. Valiron. Sur quelques propriétés des fonctions algébroides // Compt. Rend. Math. 189, 824-826 (1929).

3. N. Baganas. Sur les valeurs algébriques dune fonctions algebroldes et les intégrales pseudo-abelinnes // Annales Ecole Norm. Sup. Ser. 3. 66, 161-208 (1949).

4. H.S. Gopalkrishna, S.S. Bhooanurmath. Uniqueness theorems for meromorphic functions // Math. Scand. 39, 125-130 (1976).

5. Lahiri Indrajit, Pal Rupa. A note on Nevanlinna's five value theorerm // Bull. Korean Math. Soc. 52:2, 345-350 (2015). 
6. Yu-Zan He and Ye-Zhou Li. Some results on algebroid functions // Comp. Variab. Ellip. Equat. 43:3-4, 299-313 (2001).

7. S. Daochun, G. Zongsheng. On the operation of algebroid functions// Acta Math. Sci. 30:1, 247$256(2010)$.

8. S. Daochun, G. Zongsheng. Value disribution theory of algebroid functions. Science Press, Beijing (2014).

9. Yu-Zan He and Xiu-Zhi Xiao. Algebroid functions and Ordinarry Difference Equations. Science Press, Beijing (1988).

10. S. Daochun, G. Zongsheng. Theorems for algebroid functions // Acta Math. Sinica. 49:5, 1-6 (2006).

11. Y. Hongxun. On the multiple values and uniqueness of algebroid functions // Chinese J. Eng. Math. 8, 1-8 (1991).

12. W.K. Hayman. Meromorphic functions. Oxford University Press, Oxford (1964).

13. F. Minglang. Unicity theorem for algebroid functions // Acta. Math. Sinica. 3:6, 217-222 (1993).

14. Pingyuan Zhang, Peichu Hu. On uniqueness for algebroid functions of finite order // Acta. Math. Sinica. 35:3, 630-638 (2015).

15. G.S. Prokoporich. Fix-points of meromorphic or entire functions // Ukrainian Math. J. 25:2, 248-260 (1973).

16. Z. Qingcai. Uniqueness of algebroid functions // Math. Pract. Theory. 43:1, 183-187 (2003).

17. Cao Tingbin, Yi Hongxun. On the uniqueness theory of algebroid functions // Southest Asian Bull. Math. 33:1, 25-39 (2009).

18. Zu-Xing Xuan, ZongG-Sheng Gao. Uniqueness theorems for algebroid functions // Compl. Variab. Ellipt. Equat. 51:7, 701-712 (2006).

19. C.C. Yang, H.X. Yi. Uniqueness theory of meromorphic functions. Math. Appl. 557. Kluwer Academic Publishers, Dordrecht (2003).

20. H.X. Yi, The multiple values of meromorphic functions and uniqueness // Chinese Ann. Math. Ser. A. 10:4, 421-427 (1989).

21. R.S. Dyavanal, Ashok Rathod. Some generalisation of Nevanlinna's five-value theorem algebroid functions on annuli // Asian J. Math. Comp. Resear. 20:2, 85-95 (2017).

22. R.S. Dyavanal, Ashok Rathod. Nevanlinna's five-value heorem for derivatives of meromorphic functions sharing values on annuli // Asian J. Math. Comp. Resear. 20:1, 13-21 (2017).

23. R.S. Dyavanal, Ashok Rathod, Unicity theorem for algebroid functions related to multiple values and derivatives on annuli // Int. J. Fuzzy Math. Arch. 13:1, 25-39 (2017).

24. Ashok Rathod. Several uniqueness theorems for algebroid functions // J. Anal. 25:2, 203-213 (2017).

25. Ashok Rathod. The multiple values of algebroid functions and uniqueness // Asian J. Math. Comp. Resear. 14:2, 150-157 (2016).

26. Ashok Rathod. The multiple values of algebroid functions and uniqueness on annuli // Konoralf J. Math. 5:2, 216-227 (2017).

27. Ashok Rathod. On the deficiencies of algebriod functions and their differential polynomials // J. Basic Appl. Resear. Int. 1:1, 1-11 (2016).

Ashok Rathod,

Department of Mathematics,

Karnatak University,

Dharwad-580003,India.

E-mail: ashokmrmaths@gmail.com 